\title{
New hard X-ray emitters discovered by BAT
}

\author{
G. Cusumano* ${ }^{a}$ V. La Parola ${ }^{a}$, C. Ferrigno ${ }^{a b c}$, V. Mangano ${ }^{a}$, A. Segreto ${ }^{a}, \mathbf{P}$. \\ Romano $^{a}$, S. Campana ${ }^{e}$, G. Chincarini ${ }^{e f}$, G. Tagliaferri ${ }^{e}$, P. Giommi ${ }^{g}$ \\ ${ }^{a}$ INAF, Istituto di Astrofisica Spaziale e Fisica Cosmica, Via U. La Malfa 153 I-90146 Palermo \\ Italy \\ ${ }^{b}$ Abt. Astronomie und Astrophysik Tubingen (IAAT), Sand 1, 72076 Tubingen, Germany \\ c ISDC Data Centre for Astrophysics, Chemin d'Acogia 16, CH-1290 Versoix, Switzerland \\ e INAF, Osservatorio Astronomico di Brera, via E. Bianchi 46 I-23807 Merate Italy \\ ${ }^{f}$ Università degli studi di Milano-Bicocca, Dipartimento di Fisica, Piazza delle Scienze 3, \\ I-20126 Milan, Italy \\ ${ }^{g}$ ASI Science Data Center via Galileo Galilei I-00044 Frascati Italy \\ E-mail: cusumanodifc.inaf.it
}

We have analyzed the BAT hard X-ray survey data of the first 39 months of the Swift mission. To this end we developed a dedicated software based on a FFT algorithm that performs data reduction, background subtraction, mosaicking and source detection on the BAT survey data. This software is completely independent from the one developed by the Swift-BAT team. The large BAT field of view, the large geometrical area, and the Swift pointing strategy have allowed to obtain an unprecedented, very sensitive and quite uniform sky coverage. We analyzed the BAT dataset in three energy bands (14-150 keV, 14-30 keV, 14-70 keV) obtaining a list of 968 detections above a significance threshold of 4.8 standard deviations. The identification of the source counterparts was pursued using three strategies: cross-correlation with published hard Xray catalogues, analysis of field observations of soft X-ray instruments, cross-correlation with the Simbad databases. We derived a catalogue of 748 identified sources, of which $\sim 65 \%$ are extragalactic, $\sim 25 \%$ are Galactic objecs, $\sim 10 \%$ are already known X-ray or gamma ray emitters whose nature has not been determined yet.

7th INTEGRAL Workshop

September 8-11 2008

Copenhagen, Denmark

\footnotetext{
*Speaker.
} 


\section{Introduction}

The Burst Alert Telescope (BAT; [3]) on board the Swift observatory [5], with its large field of view $\left(100^{\circ} \times 60^{\circ}\right.$ half coded) and large detector area (a factor 2 greater than IBIS/ISGRI) offers the opportunity for a large increase of the sample of objects that contribute to the luminosity of the sky in the hard X-rays allowing for a substantial improvement of our knowledge of the AGN and of the cosmic hard X-ray background. The first results of the BAT survey have been presented in [8, 1, 2, 13]. The latter presents a catalogue of sources detected in the first 9 months of the BAT survey data, identifying 154 extragalactic sources (129 at $\left.|b|>15^{\circ}\right)$.

In order to fully expoit the BAT survey archive, we developed the BATIMAGER [11], a software independent from the one developed by the Swift-BAT team ${ }^{1}$. A complete and detailed description of the software and its performance is presented in [11]. In this paper we present the results obtained from the analysis of 39 months of BAT sky survey. More details on the method and results of the project can be found in also these proceeding in [12, 6, 7, 9]

\section{Detection strategy}

We have created all-sky maps in three energy bands: $14-150 \mathrm{keV}, 14-70 \mathrm{keV}, 14-30 \mathrm{keV}$. The source detection in the all-sky map is performed by searching for local excesses in the significance map above the absolute value of the local lowest negative excess. The source position and its peak significance are then calculated with a two dimensional Gaussian fit restricted within a region of a few pixels where the excess dominates over the noise distribution. Only detections with peak significance greater than 4.8 standard deviations are included in our list of detected sources. This threshold represents the optimal value that maximizes the number of detectable sources, maintaining at the same time an acceptable number of spurious detections. The resulting catalogues (one for each of the three energy bands) have been cross-corraleted (nearest match within the BAT error circle) in a single catalogue. We obtain a final number of 967 source candidates above our threshold in at least one of the three energy bands.

The identification of the counterpart of the BAT detections was performed following three different strategies.

a- The position of each of the 968 detected excess was cross-checked (nearest match within the BAT error circle) with the coordinates of the sources included in the INTEGRAL General Reference Catalogue $^{2}$ (v. 27), that contains 1652 X-ray emitters, and with the coordinates of the counterpart of the 48 new identifications of BAT sources already published [8, 13, 1, 2] and not included in the above catalogue. We accepted as counterpart sources candidates that were within $R=8.4^{\prime}$ from the BAT position (4 standard deviations error circle, [11]). With this method we obtain 484 identifications, 318 with $|b|>5^{\circ}$.

b- We have searched for observations from Swift/XRT containing the remaining (484) unidentified excess in their field. We found Swift/XRT observations for 167 BAT source candidates. Source detection inside these X-ray images was performed using XIMAGE (v4.4). When a source was detected inside a 6.3 arcmin error circle (99.7\% confidence level) we first checked for its hardness

\footnotetext{
${ }^{1}$ http://heasarc.gsfc.nasa.gov/docs/swift/analysis/

${ }^{2}$ http://isdc.unige.ch/?Data+catalogs
} 
ratio in the $0.3-10 \mathrm{keV}$ range (with $3 \mathrm{keV}$ as a common boundary of the two ratio bands) and for its count rate above $3 \mathrm{keV}$. We identified a source as the counterpart of the BAT detections requiring at least one of the following conditions to be satisfied: hardness ratio $>0.5$, count rate above $3 \mathrm{keV}>5 \times 10^{-3} \mathrm{c} \mathrm{s}^{-1}$. In the seven cases where two candidates, satisfying at least one of the threshold conditions, were found inside the BAT error circle, we chose as counterpart the source nearest to the BAT position. We also searched for field observations with other X-ray instruments (XMM-Newton, Chandra, BeppoSAX), finding 21 identifications, out of 26 pointings. With this method we identified 172 source counterparts.

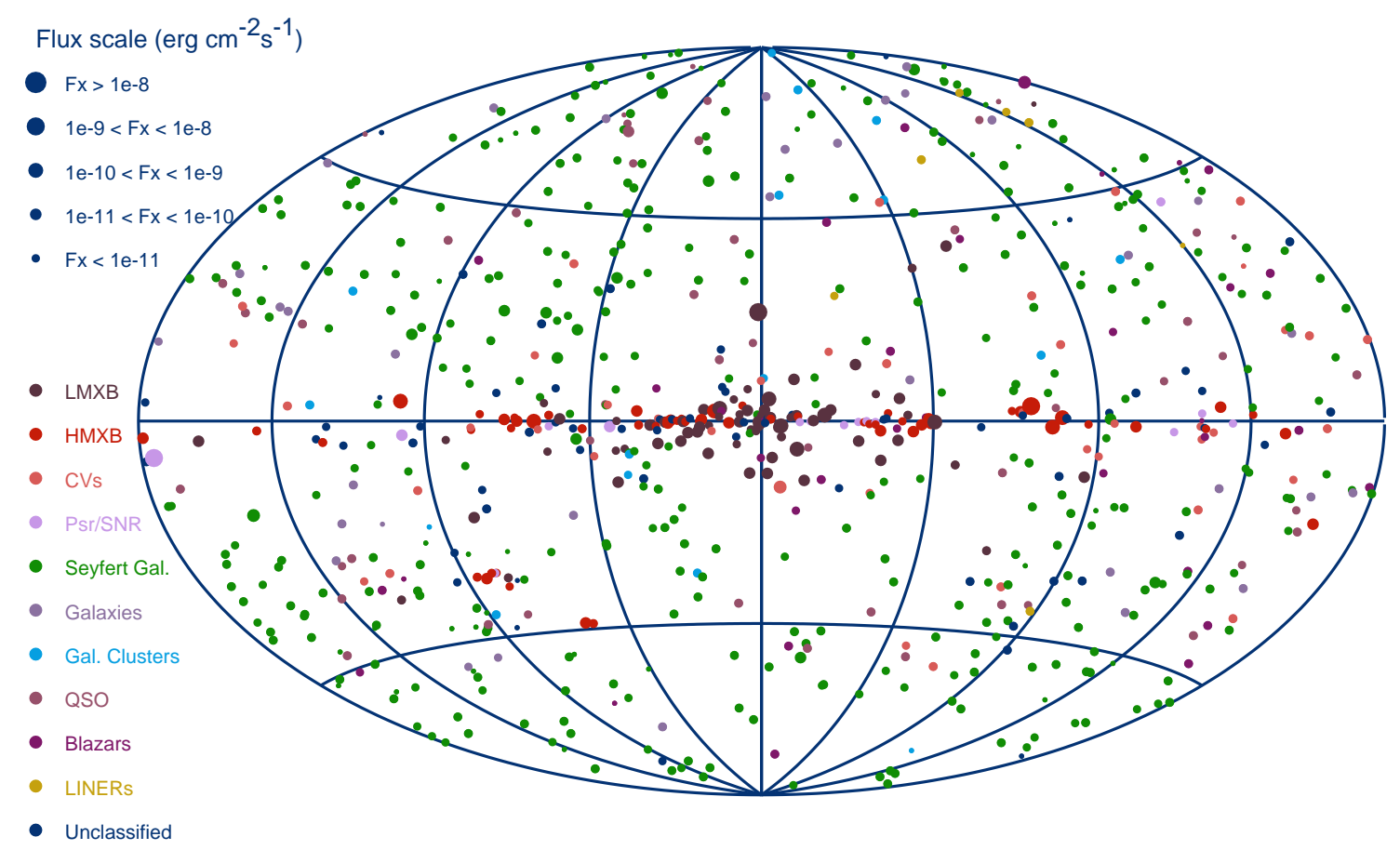

Figure 1: Map of the 748 sources identified in the BAT survey data. Different colors denote different object classes, as detailed in the legend. The size of the symbol is proportional to the source flux in the 14-150 keV band.

c- For the remaining unidentified sky map excesses (312) we searched for spatial coincidence inside an error circle of 4.2 arcmins radius (90\% confidence level, [11]) with sources included in the Simbad catalogues. The size of the search radius was fixed to $4.2 \mathrm{arcmin}$ in order to have a negligible number of spurious identifications (see below). We restrict our search to the following Simbad object classes: Cataclysmic variable (CV), High mass X-ray binaries (HXB), Low mass X-ray binaries (LXB), Seyfert 1 (Sy1), Seyfert 2 (Sy2), Blazar and BlLac (Bla,BLL), LINERs (LIN), for a total of 22425 objects in the Simbad database. This strategy allows us to identify 91 detections, with only one source at low Galactic latitude $\left(|b|>5^{\circ}\right)$.

The number of expected spurious identifications for all three strategy has been evaluated negligible: $\sim 6$ out of 748 identifications.

A set of 220 detections could not be associated with a counterpart. These source candidates have detection significance between 4.8 and 14 standard deviations and flux in the 14-150 keV 
band between $6.7 \times 10^{-12}$ and $2.7 \times 10^{-11} \mathrm{erg} \mathrm{s}^{-1} \mathrm{~cm}^{-2}$. Out of 220 sources 34 are detected in all enegy bands, 72 only in two energy bands, 201 are located above the Galactic plane $\left(|b|>5^{\circ}\right)$ and are likely extragalactic objects. These candidate sources will be object of a follow-up campaign with Swift-XRT in the future.
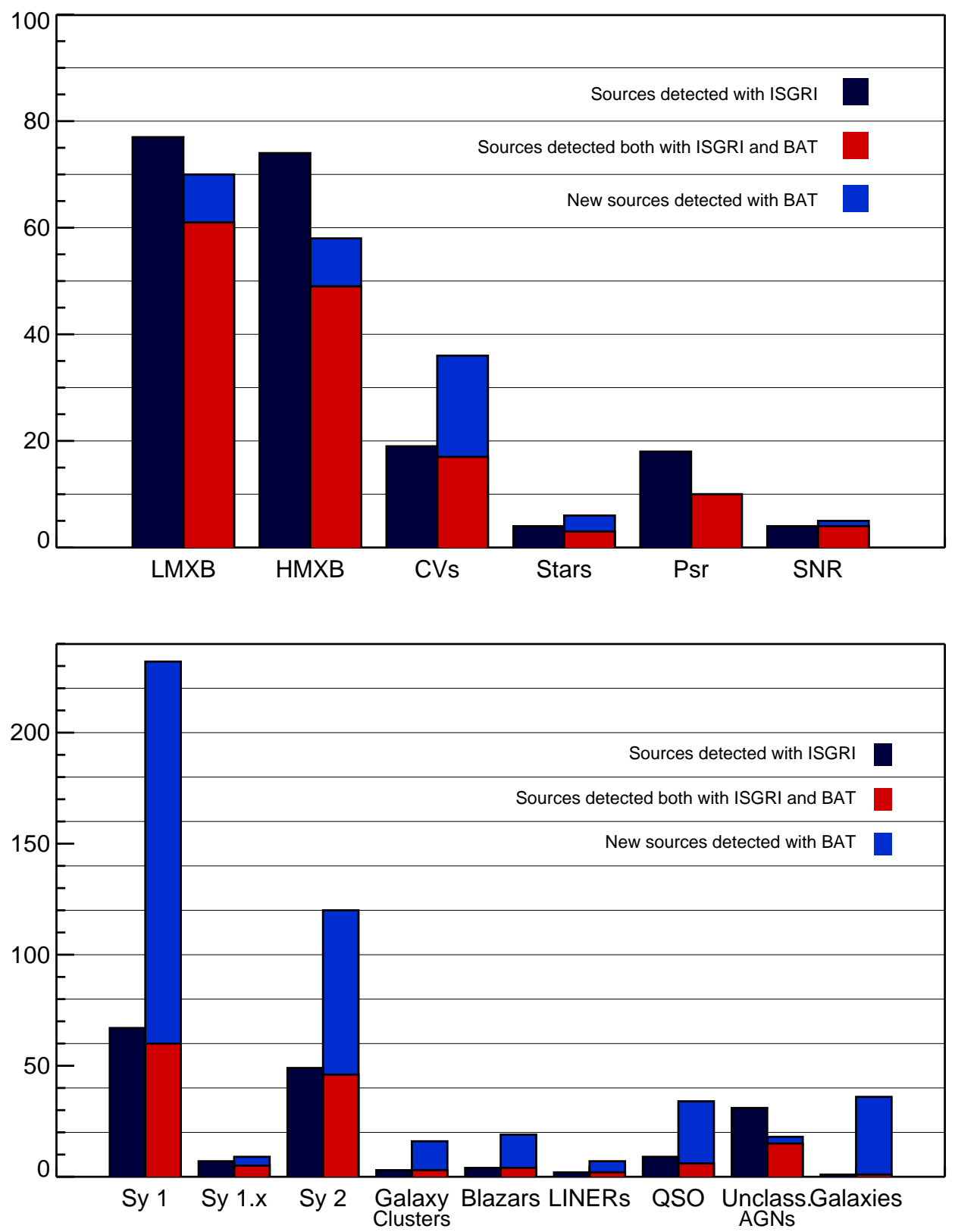

Figure 2: Comparison between the sources in our catalogue and those reported in the third ISGRI catalogue [Њ. Top: Galactic sources. Bottom: Extragalactic sources.

Table 1 details the distribution of the sources in our catalogue among different object classes: $\sim 65 \%$ of the catalogue is composed of extragalactic objects, $\sim 25 \%$ are galactic objects, $\sim 10 \%$ are already known X-ray or gamma ray emitters whose nature candidate sources will be object of 


\begin{tabular}{lrr}
\hline \hline Class & \# of sources & \% in the Catalog \\
\hline LXB & 70 & 9.4 \\
HXB & 58 & 7.8 \\
Pulsars & 10 & 1.3 \\
SN/SNR & 5 & 0.7 \\
Cataclysmic variables & 36 & 4.8 \\
Stars & 6 & 0.8 \\
\hline Galactic (total) & 185 & 24.8 \\
\hline Seyfert 1 galaxies & 232 & 31.0 \\
Seyfert 1.X galaxies & 9 & 1.2 \\
Seyfert 2 galaxies & 120 & 16.0 \\
LINERs & 7 & 0.9 \\
QSO & 34 & 4.5 \\
Blazars & 19 & 2.5 \\
Galaxy clusters & 16 & 2.1 \\
Normal galaxies & 36 & 4.8 \\
Unclassified AGN & 18 & 2.4 \\
\hline Extragalactic (total) & 491 & 65.4 \\
\hline Other types & 72 & 9.8 \\
\hline
\end{tabular}

Table 1: Classification of the known sources detected in the BAT survey. Other types includes all sources that have a catalogued counterpart but have not been classified yet.

a follow-up campaign with Swift-XRT in the future. is still to be determined. Figure 11 shows the distribution of all the sources in our catalogue, colour-coded according to the type classification, with the size of the symbol proportional to the 14-150 keV flux (for those sources not detected in the 14-150 keV band the flux in the widest band of detection has been extrapolated into the 14-150 $\mathrm{keV}$ range using a Crab-like spectrum). Most of detected sources have a flux below $1 \times 10^{-10} \mathrm{erg}$ $\mathrm{s}^{-1} \mathrm{~cm}^{-2}$ and are located outside the Galactic plane.

We have compared this distribution with the list of IBIS/ISGRI sources in the INTEGRAL General Reference Catalog (v 27), that includes also the 3rd ISGRI catalog of [4] and detections from the AGN luminosity functions of [10]. The results are plotted in Figure 2. We find a significant increase in the number of detected cataclysmic variables, and a dramatic improvement in the detection of extragalactic objects, both in the nearby Universe (normal galaxies, LINERs) and at higher distances (Seyfert galaxies, QSO, clusters of galaxies). This can be explained with the different pointing strategy of the two instruments and with the much larger field of view of the BAT, that allows a much uniform coverage of the entire sky.

\section{Summary}

The large BAT field of view, the large geometrical area, and the Swift pointing strategy have allowed to obtain an unprecedented, very sensitive and quite uniform sky coverage that has provided a significant increase of sources detected in the hard X-ray energy band (> $10 \mathrm{keV}$ ). 
We have derived a catalogue of 748 identified sources detected above a significance threshold of 4.8 standard deviations.

The extragalactic sources represents $\sim 65 \%$ of our catalogue (491 objects), $\sim 25 \%$ (181 sources) are Galactic objecs, $\sim 10 \%$ (73 sources) are already known X-ray or gamma ray emitters whose nature is still to be determined. Compared with the 3rd ISGRI catalogue [4], we identify 172 more Seyfert galaxies, 35 more normal galaxies, 13 more galaxy clusters, 28 more QSOs, 15 more Blazars and 5 more LINERs.

Among the Galactic sources we significantly increase the number of cataclysmic variables detected in the hard X-ray band (19 new objects). We also detect $18 \mathrm{X}$-ray binaries that are not included in the ISGRI catalogue, even though the total number X-ray binaries we detect is lower than the sample included in the IBIS/ISGRI catalogue.

The list of all identified hard X-ray emitters is going to be published in electronic form at http://www.pa.iasf.cnr.it/ cusumano/PalermoBatSurveyCatalogue.

\section{References}

[1] Ajello, M., Greiner, J., Kanbach, G., Rau, A., Strong, A. W., \& Kennea, J. A., BAT X-Ray Survey. I. Methodology and X-Ray Identification, 2008, ApJ, 678, 102

[2] Ajello, M., et al. The Swift BAT X-Ray Survey. III. X-Ray Spectra and Statistical Properties, 2008, ApJ, 673, 96

[3] Barthelmy, S. D., et al., The Burst Alert Telescope (BAT) on the SWIFT Midex Mission, 2005, Space Science Reviews, 120, 143

[4] Bird, A. J., et al.The Third IBIS/ISGRI Soft Gamma-Ray Survey Catalog, 2007,ApJ Supplement, 170, 175

[5] Gehrels, N., et al. The Swift Gamma-Ray Burst Mission, 2004, ApJ, 611, 1005

[6] La Parola, V. et al.Detection of ISGRI sources in 3 years of BAT all sky survey, 2009, in proceeding of 7th INTEGRAL Workshop, PoS (Integral08) 132

[7] Mangano, V. et al. Hard X-ray search for unidentified EGRET sources in the BAT and ISGRI survey archives, 2009, in proceeding of 7th INTEGRAL Workshop, PoS (Integral08) 133

[8] Markwardt, C. B., Tueller, J., Skinner, G. K., Gehrels, N., Barthelmy, S. D., \& Mushotzky, R. F. The Swift/BAT High-Latitude Survey: First Results, 2005, ApJ Letters, 633, L77

[9] Sbarufatti, B. et al.Search for Roma-BZCAT Blazars in the Palermo BAT Survey archive, 2009, in proceeding of 7th INTEGRAL Workshop, PoS (Integral08) 069

[10] Sazonov, S., Revnivtsev, M., Krivonos, R., Churazov, E., \& Sunyaev, R. Hard X-ray luminosity function and absorption distribution of nearby AGN: INTEGRAL all-sky survey, 2007, A\&A, 462, 57

[11] Segreto A., Cusumano G., Ferrigno C., La Parola V., Mangano V., Mineo T., Romano P. The Palermo Swift-BAT Survey. I. Methodology 2009, A\&A, submitted

[12] Segreto, A. et al. Hard X-ray surveys with coded mask telescopes, 2009, in proceeding of 7th INTEGRAL Workshop, PoS (Integral08)131

[13] Tueller, J., Mushotzky, R. F., Barthelmy, S., Cannizzo, J. K., Gehrels, N., Markwardt, C. B., Skinner, G. K., \& Winter, L. M. Swift BAT Survey of AGNs, 2008, ApJ, 681, 113 